AperTO - Archivio Istituzionale Open Access dell'Università di Torino

\title{
Elective Nephron Sparing Surgery Decreases Other Cause Mortality Relative to Radical Nephrectomy Only in Specific Subgroups of Patients with Renal Cell Carcinoma
}

\section{This is the author's manuscript}

Original Citation:

Availability:

This version is available http://hdl.handle.net/2318/1623648

since 2017-11-30T19:04:02Z

Published version:

DOI:10.1016/j.juro.2016.04.093

Terms of use:

Open Access

Anyone can freely access the full text of works made available as "Open Access". Works made available under a Creative Commons license can be used according to the terms and conditions of said license. Use of all other works requires consent of the right holder (author or publisher) if not exempted from copyright protection by the applicable law. 
This Accepted Author Manuscript (AAM) is copyrighted and published by Elsevier. It is posted here by agreement between Elsevier and the University of Turin. Changes resulting from the publishing process - such as editing, corrections, structural formatting, and other quality control mechanisms - may not be reflected in this version of the text. The definitive version of the text was subsequently published in THE JOURNAL OF UROLOGY, 196 (4), 2016, 10.1016/j.juro.2016.04.093.

You may download, copy and otherwise use the AAM for non-commercial purposes provided that your license is limited by the following restrictions:

(1) You may use this AAM for non-commercial purposes only under the terms of the CC-BY-NC-ND license.

(2) The integrity of the work and identification of the author, copyright owner, and publisher must be preserved in any copy.

(3) You must attribute this AAM in the following format: Creative Commons BY-NC-ND license (http://creativecommons.org/licenses/by-nc-nd/4.0/deed.en), 10.1016/j.juro.2016.04.093

The publisher's version is available at:

http://linkinghub.elsevier.com/retrieve/pii/S0022534716305080

When citing, please refer to the published version.

Link to this full text:

http://hdl.handle.net/ 


\title{
Elective Nephron Sparing Surgery Decreases Other Cause Mortality Relative to Radical Nephrectomy Only in Specific Subgroups of Patients with Renal Cell Carcinoma
}

\author{
Larcher $A^{1}$, Capitanio $U^{2}$, Terrone $C^{3}$, Volpe $A^{3}$, De Angelis $P^{3}$, Dehó $F^{1}$, Fossati $N^{1}$, Dell'Oglio $P^{1}$, Antonelli $A^{4}$, \\ Furlan $\mathrm{M}^{4}$, Simeone $\mathrm{C}^{4}$, Serni $\mathrm{S}^{5}$, Carini $\mathrm{M}^{5}$, Minervini $\mathrm{A}^{5}$, Fiori $\mathrm{C}^{6}$, Porpiglia $\mathrm{F}^{6}$, Briganti $\mathrm{A}^{1}$, Montorsi $\mathrm{F}^{1}$, \\ Bertini $R^{1}$.
}

\section{Purpose}

There is no consensus regarding a protective effect on mortality due to a cause other than cancer in patients treated with elective nephron sparing surgery relative to their radical nephrectomy counterparts. We test whether the protective effect of nephron sparing surgery relative to radical nephrectomy is universal or present in specific subgroups of patients.

\section{Materials and Methods}

A collaborative database of 5 institutions was queried to evaluate 1,783 patients without chronic kidney disease diagnosed with a clinical T1 renal mass that was treated with nephron sparing surgery or radical nephrectomy. Multivariable Cox regression analysis was done to assess the impact of surgery type (nephron sparing surgery vs radical nephrectomy) on other cause mortality after adjustment for patient and cancer characteristics. Interaction terms were used to test the hypothesis that the impact of surgery type varies according to specific subcohorts of patients.

\section{Results}

Ten-year other cause mortality-free survival rates were $90 \%$ and $88 \%$ after nephron sparing surgery and radical nephrectomy, respectively. In the overall population radical nephrectomy was not associated with an increased risk of other cause mortality on multivariable analysis compared to nephron sparing surgery (HR 0.91, 95\% CI 0.6-1.38, p = 0.6). However, radical nephrectomy increased the risk of other cause mortality according to the increasing baseline Charlson comorbidity index (interaction test $\mathrm{p}=0.0008$ ). For example, in a patient with a Charlson comorbidity index of 4 the probability of 10 -year other cause mortality-free survival was $86 \%$ after nephron sparing surgery and $60 \%$ after radical nephrectomy.

\section{Conclusions}

Elective nephron sparing surgery does not improve other cause survival relative to radical nephrectomy consistently in all patients with kidney cancer. Patients who are more ill with relevant comorbidities are those who benefit the most from nephron sparing surgery in terms of other cause mortality.

\section{Key Words}

- kidney; 
- carcinoma, renal cell;

- nephrectomy;

- cause of death;

- comorbidity

\section{Abbreviations and Acronyms}

- ACM, all cause mortality;

- BMI, body mass index;

- CCI, Charlson comorbidity index;

- EORTC, European Organisation for Research and Treatment of Cancer;

- NSS, nephron sparing surgery;

- OCM, other cause mortality;

- $\mathrm{RN}$, radical nephrectomy

Nephron sparing surgery represents the standard of care for patients diagnosed with a cT1a renal mass. ${ }^{1,2,3}$ and 4 Similarly, NSS should be preferred over RN when technically feasible in patients diagnosed with a cT1b renal mass. ${ }^{1,2}$ and 4 The rationale of these recommendations in favor of NSS is to decrease the risk of chronic kidney disease $e^{5,6 \text { and } 7}$ as well as the risk of cardiovascular events $^{8 \text { and } 9}$ while maintaining similar cancer control.

However, the controlled comparison of NSS vs RN presented in the EORTC 30904 trial showed no difference with respect to cancer specific mortality but lower ACM after RN, ${ }^{10}$ implying a paradox advantage with respect to OCM after RN. Methodological limitations of this trial have been used to justify such a counterintuitive result and prompted further observational investigations that revealed

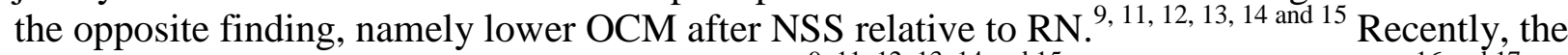
inherent risk of bias in such observational studies ${ }^{9,11,12,13,14 \text { and } 15}$ has been highlighted. ${ }^{16}$ and 17 It was postulated that if present, a beneficial effect of NSS on OCM has yet to be proved. ${ }^{18}$

In this light the aim of the study was to examine the impact of surgery type, namely NSS vs RN, on the risk of OCM in a large multi-institutional study that allowed for adjustment for a detailed panel of intrinsic confounders such as BMI, CCI, and the presence and type of hypertension or diabetes. Our hypothesis stated that NSS might be associated with a lower risk of OCM and such a benefit might be more evident in specific subgroups of patients.

\section{Materials and Methods}

\section{Study Population}

The current study relied on a collaborative database collected from 5 European tertiary care centers. Patients with a primary diagnosis of nonmetastatic clinical T1 unilateral kidney cancer without a baseline condition of chronic kidney disease treated with NSS or RN between 1984 and 2010 were included in analysis.

\section{Outcome}

The outcome of the study was OCM, defined as mortality due to a cause other than kidney cancer. Cause of death was defined according to death certificates.

\section{Covariates}


Covariates consisted of age at diagnosis, BMI, gender (male vs female), CCI, ${ }^{19}$ hypertension (no hypertension vs hypertension controlled by therapy vs hypertension uncontrolled by therapy), diabetes (present vs absent), clinical tumor size (based on preoperative imaging and defined as the greatest tumor diameter in $\mathrm{mm}$ ) and year of surgery.

\section{Statistical Analyses}

Statistical analyses, and reporting and interpreting the results were done according to established guidelines ${ }^{20}$ and consisted of 4 steps. 1) The median and IQR or frequency and proportion are reported for continuous or categorical variables, respectively. The Mann-Whitney and chi-square tests were applied to compare the statistical significance of differences in the distribution of continuous or categorical variables, respectively. The Kaplan-Meier method was used to describe 10-year OCM-free survival rates.

2) Multivariable Cox regression analysis was done to assess the impact of surgery type on OCM after adjustment for all available covariates. The nonlinear nature of the relationship between each continuous predictor and the outcome was assessed by modeling each individual variable as a restricted cubic spline. However, no evidence of nonlinearity was recorded for each continuous term (each $\mathrm{p}>0.05)$.

3) The hypothesis that the impact of surgery type was different by select patient subgroups was tested using an interaction term between treatment type (NSS vs RN) and each individual covariate.

4) Cox regression derived coefficients were used to estimate the 10-year OCM-free survival probability. The locally weighted scatterplot smoothing method ${ }^{21}$ was used to graphically explore the probability of 10-year OCM-free survival after NSS or RN according to patient baseline characteristics (eg CCI) in compliance with established methodology. ${ }^{22}$ and 23

All statistical tests were performed using RStudio ${ }^{\circledR}$, version 0.98 for $\mathrm{R}$, version 3.0.2 (https://www.r-project.org/foundation/) with the packages Hmisc, stats, rms and graphics. All tests were 2 -sided with significance considered at $\mathrm{p}<0.05$.

\section{Results}

\section{Patient Characteristics}

Overall, 1,783 patients were included in study (table 1). Patients were treated with NSS (56\% or 993) or RN (44\% or 790). Patients treated with NSS were more frequently diagnosed with CCI 0 or greater ( $48 \%$ vs $39 \%, p<0.0001)$, any kind of hypertension ( $37 \%$ vs $29 \%, p=0.0007)$, diabetes $(10 \%$ vs $6 \%, \mathrm{p}=0.02)$ and smaller tumors (median clinical size 30 vs $47 \mathrm{~mm}, \mathrm{p}<0.0001)$.

Table 1.

Descriptive characteristics of patients treated with NSS or RN for cT1 kidney cancer

No. pts $(\%)$
Median age (IQR)

No. gender $(\%)$ :
NSS RN p Value

993 (56) $790(44) \quad-$

61 (51-68) 61 (53-68) 0.2 


\begin{tabular}{|c|c|c|c|}
\hline M & $331(33)$ & $281(36)$ & \\
\hline $\mathrm{F}$ & $662(67)$ & $509(64)$ & \\
\hline Median kg/m2 BMI (IQR) & $26(24-28)$ & $26(24-28)$ & 0.3 \\
\hline No. CCI (\%): & & & $<0.0001$ \\
\hline 0 & $516(52)$ & $484(61)$ & \\
\hline 1 & $165(17)$ & $144(18)$ & \\
\hline 2 & $197(20)$ & $99(13)$ & \\
\hline 3 & $63(6)$ & $35(4)$ & \\
\hline 4 or Greater & $52(5)$ & $28(4)$ & \\
\hline No. hypertension (\%): & & & 0.0007 \\
\hline No & $623(63)$ & $563(71)$ & \\
\hline Controlled by therapy & $210(21)$ & $132(17)$ & \\
\hline Uncontrolled by therapy & $160(16)$ & $95(12)$ & \\
\hline No. diabetes (\%): & & & 0.02 \\
\hline No & $897(90)$ & $739(94)$ & \\
\hline Yes & $96(10)$ & $51(6)$ & \\
\hline Median mm clinical size (IQR) & $30(20-38)$ & $47(35-58)$ & $<0.0001$ \\
\hline No. clinical stage $(\%)$ : & & & $<0.0001$ \\
\hline cT1a & $828(83)$ & $312(39)$ & \\
\hline cT1b & $165(17)$ & $478(61)$ & \\
\hline No. diagnosis yr (\%): & & & $<0.0001$ \\
\hline 1984-1999 & 190 (19) & $429(54)$ & \\
\hline 2000-2004 & $255(26)$ & $164(21)$ & \\
\hline 2005-2010 & $548(55)$ & $197(25)$ & \\
\hline Table option & & & \\
\hline
\end{tabular}

\section{Survival}

Median followup among survivors was 70 months and 430 patients $(27 \%)$ had more than 10 years of followup. Overall, 214 deaths were recorded during the study period, including 66 due to kidney cancer and 148 due to another cause. Ten-year OCM-free survival rates were $90 \%$ after NSS and $88 \%$ after RN.

\section{Surgery Type Impact}

\section{Overall Population}

On multivariable Cox regression analysis $\mathrm{RN}$ was not associated with an increased risk of OCM when compared to NSS (HR 0.91, 95\% CI 0.6-1.38, $\mathrm{p}=0.6$, table 2). Of note, age (HR 1.09, 95\% CI 1.07-1.11, p <0.0001) and CCI (HR 1.42, 95\% CI 1.26-1.6, p=0.0001) were associated with an increased risk of OCM. Conversely, female gender (HR 0.62, 95\% CI 0.43-0.9) and year of diagnosis (HR 0.92, 95\% CI 0.88-0.95, each $\mathrm{p}=0.0001)$ were associated with a decreased risk of OCM. 
Table 2.

Multivariable Cox regression analysis predicting OCM in patients treated with NSS or RN for cT1 kidney cancer

\begin{tabular}{|c|c|c|}
\hline $\begin{array}{l}\text { Predictors } \\
\text { Surgery: }\end{array}$ & Surgery: & p Value \\
\hline NSS & 1.00 (referent) & - \\
\hline $\mathrm{RN}$ & $0.91(0.6-1.38)$ & 0.6 \\
\hline Age & $1.09(1.07-1.11)$ & $<0.0001$ \\
\hline BMI & $0.99(0.94-1.04)$ & 0.6 \\
\hline \multicolumn{3}{|l|}{ Gender: } \\
\hline M & 1.00 (referent) & - \\
\hline $\mathrm{F}$ & $0.62(0.43-0.9)$ & 0.01 \\
\hline CCI & $1.42(1.26-1.6)$ & $<0.0001$ \\
\hline \multicolumn{3}{|l|}{ Hypertension: } \\
\hline No & 1.00 (referent) & - \\
\hline Controlled by therapy & $1.06(0.65-1.71)$ & 0.8 \\
\hline Uncontrolled by therap & $0.69(0.4-1.2)$ & 0.2 \\
\hline
\end{tabular}

Diabetes:

No

Yes

Tumor size

Diagnosis yr

Table options
1.00 (referent) -

$0.61(0.32-1.17) 0.1$

$1.04(0.91-1.18) 0.5$

$0.92(0.88-0.95)<0.0001$

\section{Patient Subgroups}

The interaction tests for the hypothesis that the impact of surgery type on OCM varies according to age, BMI, gender, presence and type of hypertension, presence of diabetes, tumor size and year of diagnosis were not all statistically significant $(\mathrm{p}>0.05)$. Conversely, the interaction test for the hypothesis that the impact of surgery type on OCM varies according to patient baseline CCI was statistically significant ( $\mathrm{p}=0.0008)$. Specifically, when compared to NSS, RN increased the risk of OCM according to increasing baseline CCI. The adjusted probability of 10-year OCM-free survival after NSS or RN was plotted according to patient baseline CCI (see figure). For example, in patients with CCI 0 the probability of 10-year OCM-free survival was $95 \%$ after NSS and $94 \%$ after RN. In patients with CCI 2 the probability of 10-year OCM-free survival was $91 \%$ after NSS and $83 \%$ after RN. Finally, in patients with CCI 4 the probability of 10 -year OCM-free survival was $86 \%$ after NSS and $60 \%$ after RN. 


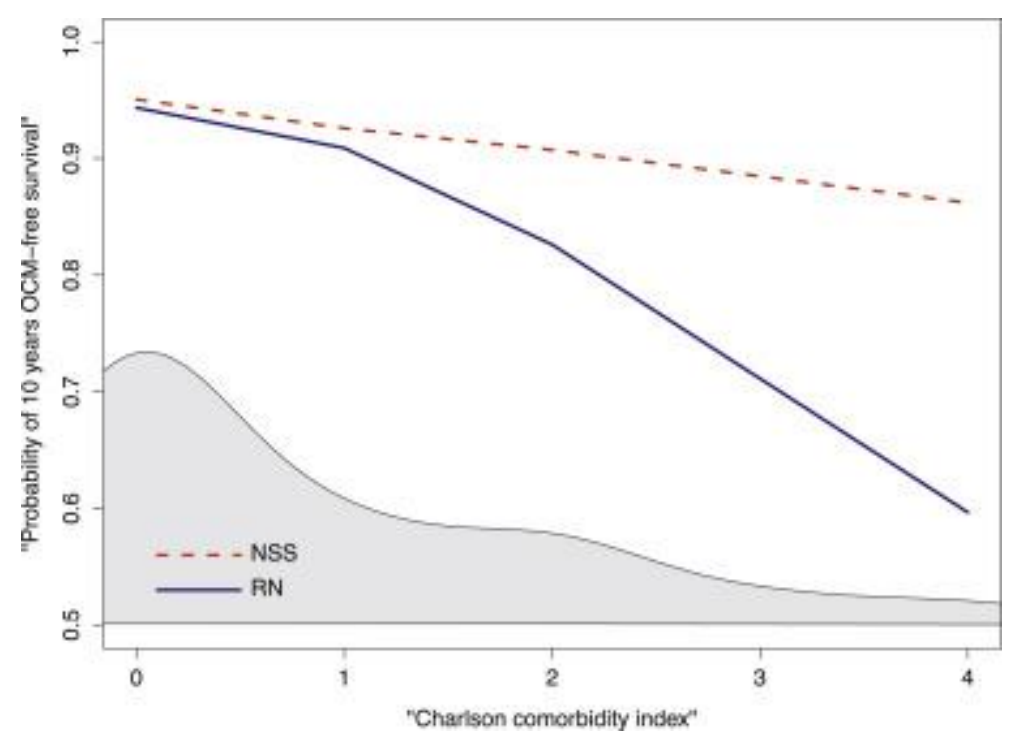

Probability of 10-year OCM-free survival after NSS or RN for cT1 kidney cancer according to baseline CCI (gray area) in 1,783 patients, including 993 treated with NSS and 790 treated with RN for cT1 kidney cancer at total of 5 European tertiary care institutions from 1984 to 2010. For example, in patients with CCI 0 probability of 10-year OCM-free survival was $95 \%$ after NSS and $94 \%$ after RN while in patients with CCI 4 probability was $86 \%$ and $60 \%$, respectively.

Figure options

\section{Discussion}

Our hypothesis stated that NSS might be associated with a lower risk of OCM relative to RN and such a benefit might be more evident in specific subgroups of patients. To test this hypothesis we relied on a large, multi-institutional kidney cancer collaborative database collected at 5 European tertiary care institutions.

Our results rejected the first statement of our hypothesis, namely that NSS might be associated with a lower risk of OCM in the overall population. It showed no difference between NSS and RN with respect to OCM after adjustment for all available potential confounders (HR 0.91, 95\% CI 0.6-1.38, $\mathrm{p}=0.6$ ). Conversely, our results confirmed the second statement of our hypothesis, namely that NSS might be associated with a lower risk of OCM in a select subgroup of patients. The interaction test for the hypothesis that the impact of surgery type on OCM varies according to patient baseline CCI showed that relative to NSS RN increased the risk of OCM according to increasing baseline CCI $(p=0.0008)$. This indicates that individuals who are more ill are those who maximally benefit from the choice of NSS (see figure). Specifically, a patient with several comorbidities (CCI 4) harbors a 10-year OCM-free survival probability of $86 \%$ after NSS and $60 \%$ after RN. Therefore, NSS should be strongly considered in patients who are more ill. On the other hand, a patient without comorbidities (CCI 0) harbors a 10-year OCM-free survival probability of 95\% after NSS and $94 \%$ after RN. Such a marginal risk reduction provides weaker arguments for selecting NSS over RN.

These findings are not negligible with respect to clinical decision making since contemporary indications ${ }^{1,2}$ and 3 supporting NSS over RN suffer from a lack of consensus about the potential OCM benefit of NSS over RN., 13 and 18 Moreover, our results represent another essential piece of 
the puzzle regarding the impact of NSS on noncancer specific survival among individuals diagnosed with a cT1 renal mass.

When compared to NSS in a randomized, controlled study, ${ }^{10} \mathrm{RN}$ resulted in similar cancer specific mortality and similar if not lower ACM rates. However, the unmet accrual end point and the important contamination rates between the trial arms bounded the clinical applicability of this finding. When compared to NSS in retrospective, observational, population based studies, ${ }^{9,} 11$, 12 and 13 RN resulted in higher ACM and OCM rates. However, more recent analyses of the same SEER (Surveillance, Epidemiology and End Results)-Medicare database showed that patients treated with RN achieved ACM-free survival comparable to that of their noncancer controls. ${ }^{16}$ Surprisingly, patients treated with NSS achieved superior ACM-free survival than noncancer controls, highlighting the risk of a biased conclusion when these surgery types are compared in a population based setting.

Notably, the current study partially reconciles such contradictory findings. Our results showed that NSS does not confer an OCM benefit in the overall population diagnosed with a cT1 renal mass but NSS translates into an OCM benefit in a select subgroup of individuals, specifically patients who are more fragile due to relevant comorbidities. This observation might explain the opposite results in the EORTC 30904 trial $^{10}$ and the Medicare based studies ${ }^{9,11,12 \text { and } 13}$ since the latter invariably relied on patients 65 years old or older and who were in consequence more ill by definition.

Moreover, the current analysis also corroborates many other findings reported in other studies with different designs. 1) The survival benefit associated with NSS was greatest in patients with a CCI of 1 or higher as also noted on subgroup analyses in a previous population based study. ${ }^{11}$

2) Prospective ${ }^{5}$ and retrospective ${ }^{6}$ and 13 studies showed superior renal function outcomes after NSS relative to RN and specifically a differential effect of NSS with respect to protection from postoperative CKD according to baseline renal function. ${ }^{24}$ Likewise, patients without comorbidities might better tolerate the renal function detriment due to RN. In consequence, in healthier individuals the survival benefit of nephron sparing is masked. On the contrary, patients with relevant comorbidities might experience a greater renal function detriment due to RN. In consequence, in individuals who are more ill the survival benefit of nephron sparing is highlighted. Similarly, NSS has been associated with lower rates of cardiovascular events than RN. ${ }^{8}$ Accordingly, it is possible that the burden of postoperative cardiovascular events is heavy enough to translate into OCM sequelae in more ill and more fragile patients, and it is lighter in healthier and more robust patients.

3) Finally, lower OCM-free survival in cases of chronic kidney disease due to medical etiology relative to surgically induced chronic kidney disease has been observed in other studies. ${ }^{25,26 \text { and } 27}$ Those findings validate the existence of a link between the general and the renal health status of the patient at baseline and the relative impact of nephron loss on the risk of OCM recorded in the current report.

It is important to remember that due to technical differences NSS is intrinsically associated with a higher risk of perioperative complication when compared to $\mathrm{RN}^{28}$ and 29 Also, the presence of comorbidities represents an established risk factor for surgical and medical complications following $\mathrm{NSS}^{23}$ Accordingly, when NSS is contemplated in patients with comorbidities, the OCM benefit demonstrated in the current study should be balanced with a potentially higher morbidity profile. Nonetheless, in the current series the overall 90-day mortality rate was extremely low at $0.3 \%$ (data not shown) and no difference was recorded after either treatment modality (unadjusted HR 6.22, p = 0.1 , data not shown). This observation corroborates previous investigations, ${ }^{28}$ and 29 suggesting that 
the reported difference with respect to perioperative morbidity does not translate into a similar difference with respect to perioperative mortality. In consequence, a higher risk of perioperative complications might be worth exchanging for a potential long-term survival benefit.

The current study has several strengths, including multi-institutional design, detailed information on the baseline cardiovascular and comorbidity profile of the patients, and inclusion of patients without baseline chronic kidney disease. However, despite its appeal and uniqueness the current study is not devoid of limitations. First and foremost, a significant limitation is related to the retrospective design of the study, which could not exclude residual confounders even after adjustment for patient and cancer characteristics. ${ }^{30}$ Recently, the risk of selection bias favoring NSS in population based studies ${ }^{16}$ was highlighted by the presence of an ACM advantage after NSS relative to RN. ${ }^{13}$ It is possible that, given the institutional nature of our report and the availability of a detailed panel of preoperative information, such biases are less relevant if not nonoperational in the current study. Accordingly, no difference was recorded after RN relative to NSS with respect to ACM (adjusted HR 1.12, $\mathrm{p}=0.5$, data not shown).

Finally, although our results demonstrated that CCI significantly affects the OCM survival benefit following NSS, our analysis did not identify which specific comorbidities should be evaluated for clinical decision making. The potential risk of a subjective CCI calculation represents an additional limitation of our comorbidities definition. In this light identifying the particular baseline health conditions that could specify the indications for NSS are yet to be investigated.

Nevertheless, despite these limitations the findings of the current study require special consideration. For the first time to our knowledge a specific group of patients who benefited from the choice of NSS over RN in terms of noncancer related survival was identified. Moreover, the information that individuals with normal preoperative renal function but who are more ill due to other comorbidities are those who maximally benefit from NSS is of the utmost importance for clinical decision making and trial planning.

\section{Conclusions}

The impact of NSS on the risk of noncancer specific mortality is not equal in all patients with normal preoperative renal function who are diagnosed with a cT1 renal mass. Specifically, in healthier patients without comorbidities NSS does not decrease the risk of OCM relative to RN. Conversely, in patients who are more ill with relevant comorbidities NSS significantly decreases the risk of OCM relative to $\mathrm{RN}$.

\section{References}

1.

○ 1

- B. Ljungberg, K. Bensalah, S. Canfield, et al.

- EAU Guidelines on renal cell carcinoma: 2014 update

- Eur Urol, 67 (2015), p. 913

- [SD-008]

2.

○ 2

- S.C. Campbell, A.C. Novick, A. Belldegrun, et al.

- Guideline for management of the clinical T1 renal mass

- J Urol, 182 (2009), p. 1271 


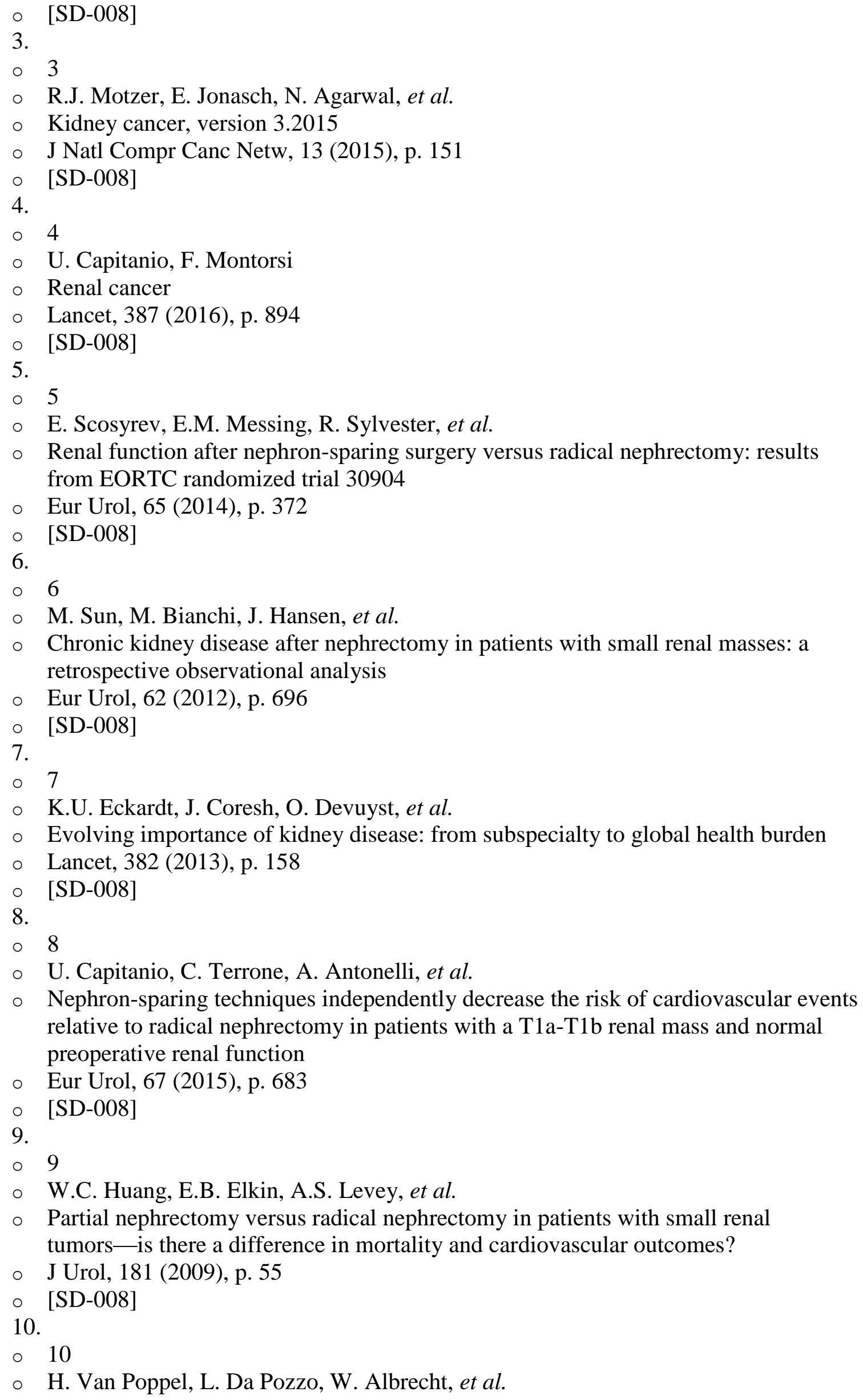


- A prospective, randomised EORTC Intergroup Phase 3 study comparing the oncologic outcome of elective nephron-sparing surgery and radical nephrectomy for low-stage renal cell carcinoma

- Eur Urol, 59 (2011), p. 543

- [SD-008]

11.

○ 11

- E.C. Norton

- Long-term survival following partial vs radical nephrectomy among older patients with early-stage kidney cancer

- JAMA, 307 (2012), p. 1629

○ [SD-008]

12.

$\circ 12$

- M. Sun, Q.D. Trinh, M. Bianchi, et al.

- A non-cancer-related survival benefit is associated with partial nephrectomy

- Eur Urol, 61 (2012), p. 725

- [SD-008]

13.

○ 13

- S.P. Kim, R.H. Thompson, S.A. Boorjian, et al.

- Comparative effectiveness for survival and renal function of partial and radical nephrectomy for localized renal tumors: a systematic review and meta-analysis

- J Urol, 188 (2012), p. 51

- [SD-008]

14.

○ 14

- K.J. Kowalczyk, T.K. Choueiri, N.D. Hevelone, et al.

- Comparative effectiveness, costs and trends in treatment of small renal masses from 2005 to 2007

BJU Int, 112 (2013), p. E273

- [SD-008]

15.

$\circ 15$

- L. Zini, P. Perrotte, U. Capitanio, et al.

- Radical versus partial nephrectomy

- Cancer, 115 (2009), p. 1465

- [SD-008]

16.

○ 16

- B. Shuch, J. Hanley, J. Lai, et al.

Overall survival advantage with partial nephrectomy: a bias of observational data?

Cancer, 119 (2013), p. 2981

[SD-008]

17.

○ 17

- A. Kutikov, M.C. Smaldone, R.G. Uzzo

- Partial versus radical nephrectomy: balancing nephrons and perioperative risk

- Eur Urol, 64 (2013), p. 607

$\circ$ [SD-008]

18. 
○ 18

- C.M. Tobert, C.B. Riedinger, B.R. Lane

- Do we know (or just believe) that partial nephrectomy leads to better survival than radical nephrectomy for renal cancer?

- World J Urol, 32 (2014), p. 573

- [SD-008]

19.

○ 19

- M.E. Charlson, P. Pompei, K.L. Ales, et al.

- A new method of classifying prognostic comorbidity in longitudinal studies: development and validation

- J Chronic Dis, 40 (1987), p. 373

- [SD-008]

20.

○ 20

- A.J. Vickers, D.D. Sjoberg

- Guidelines for reporting of statistics in European Urology

- Eur Urol, 67 (2015), p. 181

$\circ$ [SD-008]

21.

○ 21

- W.S. Cleveland

- Robust locally weighted regression and smoothing scatterplots

- J Am Stat Assoc, 74 (1979), p. 829

$\circ$ [SD-008]

22.

○ 22

- N. Fossati, Q.D. Trinh, J. Sammon, et al.

- Identifying optimal candidates for local treatment of the primary tumor among patients diagnosed with metastatic prostate cancer: a SEER-based study

- Eur Urol, 67 (2015), p. 3

○ [SD-008]

23.

$\circ 23$

- A. Larcher, N. Fossati, Z. Tian, et al.

- Prediction of complications following partial nephrectomy: implications for ablative techniques candidates

- Eur Urol, 69 (2016), p. 676

- [SD-008]

24.

○ 24

- S.L. Woldu, A.C. Weinberg, R. Korets, et al.

- Who really benefits from nephron-sparing surgery?

- Urology, 84 (2014), p. 860

- [SD-008]

25.

○ 25

- B.R. Lane, S.C. Campbell, S. Demirjian, et al.

- Surgically induced chronic kidney disease may be associated with a lower risk of progression and mortality than medical chronic kidney disease

○ J Urol, 189 (2013), p. 1649 
$\circ \quad[\mathrm{SD}-008]$

26.

○ 26

- B.R. Lane, S. Demirjian, I.H. Derweesh, et al.

- Survival and functional stability in chronic kidney disease due to surgical removal of nephrons: importance of the new baseline glomerular filtration rate

- Eur Urol, 68 (2015), p. 996

$\circ \quad[\mathrm{SD}-008]$

27.

○ 27

- D. Parker, A. Kutikov, R.G. Uzzo, et al.

- Understanding chronic kidney disease of surgical versus medical origin: the missing link to the partial versus radical nephrectomy debate?

- Eur Urol, 68 (2015), p. 1004

- [SD-008]

28.

- 28

- H. Van Poppel, L. Da Pozzo, W. Albrecht, et al.

- A prospective randomized EORTC intergroup phase 3 study comparing the complications of elective nephron-sparing surgery and radical nephrectomy for lowstage renal cell carcinoma

- Eur Urol, 51 (2007), p. 1606

- [SD-008]

29.

○ 29

- A. Becker, P. Ravi, F. Roghmann, et al.

- Laparoscopic radical nephrectomy vs laparoscopic or open partial nephrectomy for T1 renal cell carcinoma: comparison of complication rates in elderly patients during the initial phase of adoption

- Urology, 83 (2014), p. 1285

$\circ \quad$ [SD-008]

30.

○ 30

- J.J. Tomaszewski, A. Kutikov

- Retrospective comparison of cardiovascular risk in preselected patients undergoing kidney cancer surgery: reflection of reality or simply what we want to hear?

- Eur Urol, 67 (2015), p. 690

- [SD-008] 